\title{
Article
}

\section{On the oscillation of fractional differential equations via $\psi$-Hilfer fractional derivative}

\author{
Devaraj Vivek $^{1, *}$, Elsayed M. Elsayed ${ }^{2,3}$ and Kuppusamy Kanagarajan ${ }^{4}$ \\ 1 Department of Mathematics with Computer Applications, Sri Ramakrishna College of Arts and Science (Formerly \\ SNR sons College), Coimbatore-641 006, India. \\ 2 Department of Mathematics, Faculty of Science, Mansoura University, Mansoura 35516, Egypt.; \\ emmelsayed@yahoo.com \\ 3 Department of Mathematics, Faculty of Science, King Abdulaziz University,Jeddah 21589, Saudi Arabia. \\ 4 Department of Mathematics, Sri Ramakrishna Mission Vidyalaya College of Arts and Science, Coimbatore-20, India.; \\ kanagarajank@gmail.com \\ * Correspondence: peppyvivek@gmail.com
}

Received: 11 April 2019; Accepted: 26 May 2019; Published: 12 July 2019.

Abstract: In this paper, we study the oscillatory theory for fractional differential equations (FDEs) via $\psi$-Hilfer fractional derivative. Sufficient conditions are established for the oscillation of solutions FDEs.

Keywords: Caputo derivative, $\psi$-Hilfer fractional derivative, oscillation, Riemann-Liouville operator.

\section{Introduction}

$\mathbf{O}$ ver the decades, the fractional calculus has been building a great history and consolidating itself in several scientific areas such as: mathematics, physics and engineering, among others. The emergence of new fractional integrals and derivatives, makes the wide number of definitions becomes increasingly larger and clears its numerous applications. Recently, the existence of solutions of initial and boundary value problems for differential equations involving Hilfer fractional derivative has a considerable attention [1-8].

Very recently, Almeida [9] introduced a new fractional derivative named by $\psi$-fractional derivative with respect to another function, which extended the classical fractional derivative and also studied some properties like semigroup law, Taylor's Theorem and so on. Thereafter, Sousa and Oliveira $[10,11]$ initially studied a Cauchy problem for fractional ordinary differential equation with $\psi$-Hilfer operator with respect to another function, in order to unify the wide number of fractional derivatives in a single fractional operator and consequently, open a window for new applications and established a new Gronwall inequality to derive a prior bound of a solution. The authors studied the Leibniz type rule: $\psi$-Hilfer fractional operator in [12].

The oscillation theory as a part of the qualitative theory of differential equations has been developed rapidly in the last decades and there has been a great deal of work on the oscillatory behavior of integer order differential equations. However, there are only very few papers dealing with the oscillation of FDEs; see [13-15]. The study of oscillation and other qualitative properties of fractional dynamical systems such as stability, existence, and uniqueness of solutions is necessary to analyze the systems under consideration $[16,17]$.

Motivated by [18] and the aforementioned papers, we study the oscillatory theory for $\psi$-Hilfer fractional type FDEs of the form

$$
\begin{aligned}
& D_{a^{+}}^{\alpha, \beta ; \psi} x(t)+f_{1}(t, x)=w(t)+f_{2}(t, x), \\
& I_{a^{+}}^{1-\gamma ; \psi} x(t)=b_{1},
\end{aligned}
$$

where $D_{a^{+}}^{\alpha, \beta ; \psi}$ denotes the $\psi$-Hilfer fractional derivative of order $0<\alpha<1$ type $0 \leq \beta \leq 1, I_{a^{+}}^{1-\gamma ; \psi}$ is the $\psi$-Riemann-Liouville fractional integral with $\gamma=\alpha+\beta(1-\alpha)$ and $b_{1}>0$.

We assume in this paper that the functions $f_{1}, f_{2}$ and $w$ are continuous. The solution representation of (1)-(2) can be written as 


$$
x(t)=\left\{\begin{array}{l}
\frac{b_{1}(\psi(t)-\psi(a))^{\gamma-1}}{\Gamma(\gamma)} \\
+\frac{1}{\Gamma(\alpha)} \int_{a}^{t} \psi^{\prime}(s)(\psi(t)-\psi(s))^{\alpha-1}\left[w(s)+f_{2}(s, x(s))-f_{1}(s, x(s))\right] d s
\end{array} .\right.
$$

We only take those solutions which are continuous and continuable to $(a, \infty)$, and are not identically zero on any half-line $(b, \infty)$ for some $b \geq a$. The term "solution"' henceforth applies to such solutions of equations (1) or (3). A solution is said to be oscillatory if it has arbitrarily large zeros on $(0, \infty)$; otherwise, it is called nonoscillatory.

\section{Main results}

We will make use of the conditions:

$$
x f_{i}(t, x)>0 \quad(i=1,2), \quad x \neq 0, t \leq a
$$

and

$$
\left|f_{1}(t, x)\right| \geq p_{1}(t)|x|^{v}, \quad\left|f_{2}(t, x)\right| \leq p_{2}(t)|x|^{u}, \quad x \neq 0, t \geq a,
$$

where $p_{1}, p_{2} \in C\left([a, \infty], \mathbb{R}^{+}\right)$and $u, v>0$ are real numbers.

We will use the following lemma [[19], Lemma 1]

Lemma 1. For $\mathscr{X} \geq 0$ and $\mathscr{Y}>0$, we have

$$
\mathscr{X}^{\lambda}+(\lambda-1) \mathscr{Y}^{\lambda}-\lambda \mathscr{X} \mathscr{Y}^{\lambda-1} \geq 0, \quad \lambda>1
$$

and

$$
\mathscr{X}^{\lambda}+(1-\lambda) \mathscr{Y}^{\lambda}-\lambda \mathscr{X} \mathscr{Y}^{\lambda-1} \leq 0, \quad \lambda<1,
$$

where equality holds if and only if $\mathscr{X}=\mathscr{Y}$.

Now we may give our first theorem when $f_{2}=0$.

Theorem 2. Let $f_{2}=0$ and condition (4) hold. If

$$
\lim _{t \rightarrow \infty} \inf (\psi(t))^{1-\gamma} \int_{a}^{t} \psi^{\prime}(s)(\psi(t)-\psi(s))^{\alpha-1} w(s) d s=-\infty,
$$

and

$$
\lim _{t \rightarrow \infty} \sup (\psi(t))^{1-\gamma} \int_{a}^{t} \psi^{\prime}(s)(\psi(t)-\psi(s))^{\alpha-1} w(s) d s=\infty
$$

Proof. Let $x(t)$ be a non-oscillatory solution of equations (1)-(2) with $f_{2}=0$. Suppose that $T>a$ is large enough so that $x(t)>0$ for $t \leq T$.

Let $F(t)=w(t)+f_{2}(t, x(t))-f_{1}(t, x(t))$, then we see from (3) that

$$
\begin{aligned}
x(t) \leq & \frac{(\psi(t)-\psi(a))^{\gamma-1}}{\Gamma(\gamma)}\left|b_{1}\right|+\frac{1}{\Gamma(\alpha)} \int_{a}^{T} \psi^{\prime}(s)(\psi(t)-\psi(s))^{\alpha-1}|F(s)| d s \\
& +\frac{1}{\Gamma(\alpha)} \int_{a}^{T} \psi^{\prime}(s)(\psi(t)-\psi(s))^{\alpha-1} w(s) d s, \quad t \geq T,
\end{aligned}
$$


and hence

$$
\Gamma(\alpha)(\psi(t))^{1-\gamma} x(t) \leq c(T)+(\psi(t))^{1-\gamma} \int_{T}^{t} \psi^{\prime}(s)(\psi(t)-\psi(s))^{\alpha-1} w(s) d s,
$$

where,

$$
c(T)=\frac{1}{\Gamma(\gamma)}\left(\frac{\psi(T)}{\psi(T)-\psi(a)}\right)^{1-\gamma}\left|b_{1}\right|+\int_{a}^{T}\left(\frac{\psi(T)}{\psi(T)-\psi(s)}\right)^{1-\alpha}|F(s)| d s .
$$

Note that the improper integral on the right is convergent. Applying the limit inferior of both sides of inequality (11) as $t \rightarrow \infty$, we obtain a contradiction to condition (8). In case $x(t)$ is eventually negative, a similar argument leads to a contradiction with (9).

Next we have the following results.

Theorem 3. Let conditions (1)-(2) and (2) hold with $v>1$ and $u=1$. If

$$
\lim _{t \rightarrow \infty} \inf (\psi(t))^{1-\gamma} \int_{a}^{t} \psi^{\prime}(s)(\psi(t)-\psi(s))^{\alpha-1}\left[w(s)+\mathscr{H}_{v}(s)\right] d s=-\infty
$$

and

$$
\lim _{t \rightarrow \infty} \sup (\psi(t))^{1-\gamma} \int_{a}^{t} \psi^{\prime}(s)(\psi(t)-\psi(s))^{\alpha-1}\left[w(s)+\mathscr{H}_{v}(s)\right] d s=\infty
$$

where

$$
\mathscr{H}_{v}(s)=(v-1) v^{\frac{v}{(1-v)}} p_{1}^{\frac{1}{(1-v)}}(s) p_{2}^{\frac{1}{(v-1)}}(s),
$$

then every solution of equation (1) is oscillatory.

Proof. Let $x(t)$ be a nonoscillatory solution of equations (3), say, $x(t)>0$ for $r \geq T>a$. Using (5) in equation (3) with $u=1$ and $v>1$ and $t \geq T$, we find

$$
\begin{aligned}
\Gamma(\gamma)(\psi(t))^{1-\gamma} x(t) & \leq c(T)+(\psi(t))^{1-\gamma}\left[\int_{t}^{T} \psi^{\prime}(s)(\psi(t)-\psi(s))^{\alpha-1} w(s) d s\right. \\
& \left.+\int_{t}^{T} \psi^{\prime}(t)(\psi(t)-\psi(s))^{\alpha-1}\left[p_{2}(s) x(s)-p_{1}(s) x^{v}(s)\right] d s\right] .
\end{aligned}
$$

We apply (6) in Lemma 1 with

$$
\lambda=v, \mathscr{X}=p_{1}^{\frac{1}{v}} x \quad \text { and } \quad \mathscr{Y}=\left(p_{2} p_{1}^{\frac{-1}{v}} / v\right)^{\frac{1}{(v-1)}}
$$

to obtain

$$
p_{2}(t) x(t)-p_{1}(t) x^{v}(t) \leq(v-1) v^{\frac{v}{(1-v)}} p_{1}^{\frac{1}{(1-v)}}(t) p_{2}^{\frac{v}{(v-1)}}(t)
$$

Using (16) in (15), we have

$$
\Gamma(\gamma)(\psi(t))^{1-\gamma} x(t) \leq c(T)+(\psi(t))^{1-\gamma} \int_{T}^{t} \psi^{\prime}(t)[\psi(t)-\psi(s)]^{\alpha-1}\left[w(s)+\mathscr{H}_{v}(s)\right] d s, \quad t \geq T .
$$

The rest of the proof is the similar as in that of Theorem 2. 
Theorem 4. Let condition (4) and (5) hold with $v=1$ and $u<1$. If

$$
\lim _{t \rightarrow \infty} \inf (\psi(t))^{1-\gamma} \int_{a}^{t} \psi^{\prime}(s)(\psi(t)-\psi(s))^{\alpha-1}\left[w(s)+\mathscr{H}_{u}(s)\right] d s=-\infty
$$

and

$$
\lim _{t \rightarrow \infty} \sup (\psi(t))^{1-\gamma} \int_{a}^{t} \psi^{\prime}(s)(\psi(t)-\psi(s))^{\alpha-1}\left[w(s)+\mathscr{H}_{u}(s)\right] d s=\infty
$$

where,

$$
\mathscr{H}_{u}(s)=(1-u) u^{\frac{u}{(u-1)}} p_{1}^{\frac{u}{(u-1)}}(s) p_{2}^{\frac{1}{(1-u)}}(s),
$$

then every solution of equations of (1)-(2) is oscillatory.

Proof. Let $x(t)$ be a nonoscillatory solution of equations (3), say $x(t)>0$ for $t \geq a>1$. Using condition (5) in (3), with $v=1$ and $u<1$, we obtain

$$
\begin{aligned}
\Gamma(\alpha)(\psi(t))^{1-\gamma} x(t) & \leq c(T)+(\psi(t))^{1-\alpha}\left[\int_{a}^{t} \psi^{\prime}(s)(\psi(t)-\psi(s))^{\alpha-1} w(s) d s\right. \\
& \left.+\int_{a}^{t} \psi^{\prime}(s)(\psi(t)-\psi(s))^{\alpha-1}\left[p_{2}(s) x^{u}(s)-p_{1}(s) x(s)\right] d s\right]
\end{aligned}
$$

Now we use (7) in Lemma 1 with

$$
\lambda=u, \mathscr{X}=p_{2}^{\frac{1}{u}} x \quad \text { and } \quad \mathscr{Y}=\left(p_{1} p_{2}^{\frac{-1}{u}} / u\right)^{\frac{1}{(u-1)}}
$$

to get

$$
p_{2}(t) x^{u}(t)-p_{1}(t) x(t) \leq(1-u) u^{\frac{u}{(1-u)}} p_{1}^{\frac{u}{(u-1)}}(t) p_{2}^{\frac{1}{(1-u)}}(t)
$$

Using (20) in (19) then yields

$$
\Gamma(\alpha)(\psi(t))^{1-\gamma} x(t) \leq c(T)+(\psi(t))^{1-\gamma} \int_{t}^{T} \psi^{\prime}(s)(\psi(t)-\psi(s))^{\alpha-1}\left[w(s)+\mathscr{H}_{u}(s)\right] d s, \quad t \geq T .
$$

The rest of the proof is the similar as in that of Theorem 2.

Theorem 5. Let condition (4) and (5) hold with $v>1$ and $u<1$. If

$$
\lim _{t \rightarrow \infty} \inf (\psi(t))^{1-\gamma} \int_{a}^{t} \psi^{\prime}(s)(\psi(t)-\psi(s))^{\alpha-1}\left[w(s)+\mathscr{H}_{v, u}(s)\right] d s=-\infty
$$

and

$$
\lim _{t \rightarrow \infty} \sup (\psi(t))^{1-\gamma} \int_{a}^{t} \psi^{\prime}(s)(\psi(t)-\psi(s))^{\alpha-1}\left[w(s)+\mathscr{H}_{v, u}(s)\right] d s=\infty
$$

where

$$
\mathscr{H}_{v, u}(s)=(v-1) v^{\frac{v}{(1-v)}} \epsilon^{\frac{v}{(v-1)}}(s) p_{1}^{\frac{1}{(1-v)}}(s)+(1-u) u^{\frac{u}{(1-u)}} \epsilon^{\frac{u}{(u-1)}}(s) p_{2}^{\frac{1}{(1-u)}}(s)
$$

with $\epsilon \in C\left([a, \infty], \mathbb{R}^{+}\right)$, then every solution of equation (1)-(2) is oscillatory. 
Proof. Let $x(t)$ be a nonoscillatory solution of (1)-(2), say $x(t)>0$ for $t \geq T>a$. Using (5) in equation (3) one can easily write that

$$
\begin{aligned}
\Gamma(\alpha)(\psi(t))^{1-\gamma} x(t) & \geq c(T)+(\psi(t))^{1-\gamma} \int_{T}^{t} \psi^{\prime}(s)(\psi(t)-\psi(s))^{\alpha-1} w(s) d s \\
& +(\psi(t))^{1-\gamma} \int_{T}^{t} \psi^{\prime}(s)(\psi(t)-\psi(s))^{\alpha-1}\left(\epsilon(s) x(s)-p_{1}(s) x^{v}(s)\right) d s \\
& +(\psi(t))^{1-\gamma} \int_{T}^{t} \psi^{\prime}(s)(\psi(t)-\psi(s))^{\alpha-1}\left(p_{2}(s) x^{u}(s)-\epsilon(s) x(s)\right) d s, t \geq T .
\end{aligned}
$$

We may bound the term $\left(\epsilon x-p_{1} x^{v}\right)$ and $\left(p_{2} x^{u}-\epsilon x\right)$ by using inequalities (16) (with $\left.p_{2}=\epsilon\right)$ respectively; to get

$$
\Gamma(\alpha)(\psi(t))^{1-\gamma} x(t) \leq c(T)+(\psi(t))^{1-\alpha} \int_{T}^{t} \psi^{\prime}(s)(\psi(t)-\psi(s))^{\alpha-1}\left[w(s)+\mathscr{H}_{v, u}(s)\right] d s, \quad t \geq T .
$$

The rest of the proof is the similar as in that of Theorem 2.

Remark 1. The result obtained from (1) are with different nonlinearities and one can observe that the forcing term $w$ is unbounded, and its oscillatory character is inherited by the solutions.

Author Contributions: All authors contributed equally to the writing of this paper. All authors read and approved the final manuscript.

Conflicts of Interest: "The authors declare no conflict of interest."

\section{References}

[1] Furati, K. M., \& Kassim, M. D. (2012). Existence and uniqueness for a problem involving Hilfer fractional derivative. Computers \& Mathematics with Applications, 64(6), 1616-1626.

[2] Gao, Z., \& Yu, X. (2018). Existence results for BVP of a class of Hilfer fractional differential equations. Journal of Applied Mathematics and Computing, 56(1-2), 217-233.

[3] Rudolf, H. (Ed.). (2000). Applications of fractional calculus in physics. World Scientific.

[4] Vivek, D., Kanagarajan, K.,\& Elsayed, E. M. (2018). Some existence and stability results for Hilfer-fractional implicit differential equations with nonlocal conditions. Mediterranean Journal of Mathematics, 15(1), 15.

[5] Vivek, D., Kanagarajan, K., \& Elsayed, E. M. (2018). Nonlocal initial value problems for implicit differential equations with Hilfer-Hadamard fractional derivative. Nonlinear Anal Model Control, 23(3), 341-360.

[6] Vivek, D., Kanagarajan, K., \& Sivasundaram, S. (2017). Dynamics and stability results for Hilfer fractional type thermistor problem. Fractal and Fractional, 1(1), 5.

[7] Vivek, D., Kanagarajan, K., \& Sivasundaram, S. (2017). Theory and analysis of nonlinear neutral pantograph equations via Hilfer fractional derivative. Nonlinear Studies, 24(3).

[8] Vivek, D., Kanagarajan, K., \& Sivasundaram, S. (2016). Dynamics and stability of pantograph equations via Hilfer fractional derivative. Nonlinear Studies, 23(4).

[9] Almeida, R. (2017). A Caputo fractional derivative of a function with respect to another function. Communications in Nonlinear Science and Numerical Simulation, 44, 460-481.

[10] Sousa, J., \& de Oliveira, E. C. (2017). A Gronwall inequality and the Cauchy-type problem by means of $\psi$-Hilfer operator. Differential Equations and Applications, 11(1), (2019), 87-106.

[11] Sousa, J. V. D. C., \& de Oliveira, E. C. (2018). On the $\psi$-Hilfer fractional derivative. Communications in Nonlinear Science and Numerical Simulation, 60, 72-91.

[12] Sousa, J. V. D. C., \& de Oliveira, E. C. (2019). Leibniz type rule: $\psi$-Hilfer fractional operator, Communications in Nonlinear Science and Numerical Simulation, 77, 305-311.

[13] Chen, D. X. (2012). Oscillation criteria of fractional differential equations. Advances in Difference Equations, 2012(1), 33.

[14] Chen, D. X. (2013). Oscillatory behavior of a class of fractional differential equations with damping. UPB Scientific Bulletin, Series A, 75(1), 107-118.

[15] Marian, S. L., Sagayaraj, M. R., Selvam, A. G. M., \& Loganathan, M. P. (2012). Oscillation of fractional nonlinear difference equations. Mathematica Aeterna, 2(9-10), 805-813. 
[16] Khan, H., Chen, W., \& Sun, H. (2018). Analysis of positive solution and Hyersá Ulam stability for a class of singular fractional differential equations with p-Laplacian in Banach space. Mathematical Methods in the Applied Sciences, 41(9), 3430-3440.

[17] Khan, H., Tunc, C., Chen, W., \& Khan, A. (2018). Existence theorems and Hyersá Ulam stability for a class of hybrid fractional differential equations with p-Laplacian operator. J. Appl. Anal. Comput, 8(4), 1211-1226.

[18] Grace, S., Agarwal, R., Wong, P., \& Zafer, A. (2012). On the oscillation of fractional differential equations. Fractional Calculus and Applied Analysis, 15(2), 222-231.

[19] Agarwal, R. P., Grace, S. R., \& O’Regan, D. (2002). Oscillation theory for second order linear, half-linear, superlinear and sublinear dynamic equations. Springer Science \& Business Media.

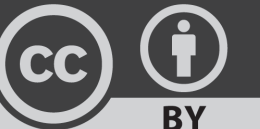

BY

(C) 2019 by the authors; licensee PSRP, Lahore, Pakistan. This article is an open access article distributed under the terms and conditions of the Creative Commons Attribution (CC-BY) license (http://creativecommons.org/licenses/by/4.0/). 